\title{
BIBLIOGRAPHY
}

1. R. F. Arens, A topology for spaces of transformations, Ann. of Math. vol. 47 (1946) pp. 480-495.

2. R. F. Arens ond J. L. Kelley, Characterizations of the space of continuous functions over a compact Hausdorff space, To be published in Trans. Amer. Math. Soc.

3. S. Banach, Theorie des operations lineaires, Warsaw, 1932.

4. G. Birkhoff, Lattice theory, Amer. Math. Soc. Colloquium Publications, vol. 25, New York, 1940.

5. S. Eilenberg, Banach space methods in topology, Ann. of Math. vol. 43 (1942) pp. 568-579.

6. I. Gelfand and A. N. Kolmogoroff, On rings of continuous functions on a topological space, C. R. (Doklady) URSS. vol. 22 (1939) pp. 11-15.

7. M. H. Stone, Applications of the theory of Boolean rings to general topology, Trans. Amer. Math. Soc. vol. 41 (1937) pp. 375-481.

8. - Topological representations of distributive lattices and Brouwerian logics, Casopis Matematiky a Fysiky vol. 67 (1937) pp. 1-25.

9. - A general theory of spectra II, Proc. Nat. Acad. Sci. U.S.A. vol. 27 (1941) pp. 83-87.

University of Chicago

\section{LINEAR TOPOLOGICAL DIVISION ALGEBRAS}

RICHARD ARENS

1. Introduction. We present a generalization of the familiar theorem of Frobenius that any finite-dimensional linear division algebra $A$ over the real number field is isomorphic to the real, the complex, or the quaternion number system. The generalization consists in replacing the hypothesis of finite dimensionality of $A$ by the weaker hypothesis that $A$ be a complete linear space with a topology in which multiplication is continuous and which is based on a countable system of convex open sets.

Previous generalizations of Frobenius' theorem have been indicated or proved by S. Mazur [6], ${ }^{1}$ G. Silov [8], and I. Gelfand [4]. These writers have generally assumed that $A$ have a norm; and only that case has been adequately considered in which scalar multiplication by complex numbers is assumed. We shall give the proof of the general case without limiting ourselves to the commutative case or to complex scalars. 1946.

Presented to the Society August 23, 1946; received by the editors November 20,

${ }^{1}$ Numbers in brackets refer to the bibliography at the end of the paper. 
In the presence of a norm, the operation of inversion, that is, the passage from $x$ to $x^{-1}$ in $A$, is easily seen to be analytic (in a sense defined in [4]) and an application of the Liouville theorem establishes that $A$ is the complex number system. In connection with nonnormed algebras one is hampered by the fact that inversion need not even be continuous on the set of nonsingular elements. In other words, that part of a topological ring which forms a multiplicative group need not be a topological group in its relative topology: a discussion of this follows in $\$ 4$. However, in complete separable metric division rings, inversion is nevertheless continuous on the set of nonzero elements, as a result of a theorem on topological groups due to D. Montgomery [7]. Because of this, an adaptation of Gelfand's method can be made which establishes our Theorem 3 .

In a section devoted to integral domains we generalize slightly a theorem of Silov [8] without being able to avoid hypothesizing a norm. We justify this limitation by exhibiting the peculiar properties of the ring $A$ of functions holomorphic on an open set. This is a complete metric convex linear algebra and is an integral domain, even in a strong topological sense. Its existence with these properties shows that Frobenius' theorem formulated in terms of integral domains does not generalize as readily as the statement above in terms of the existence of inverses. The most remarkable property of this ring of holomorphic functions is probably the fact that the set of singular elements is open and not closed as is the case in complete normed rings.

2. Topological division algebras. A topological algebra $A$ is a topological linear space in which not only addition and scalar multiplication by real numbers, but also multiplication of elements of $A$ is defined, and in such a manner as to be continuous. If scalar multiplication by complex numbers is defined as well, we speak of a complex topological algebra. We call $A$ a convex topological algebra if it is convex as a topological linear space (see [10]). If the topology of $A$ can be given by a metric structure, we call it a metric linear space; and we say $A$ is complete if it is a complete metric space. An important special case of convex complete metric algebras is presented by the so-called normed rings [4], otherwise known as Banach algebras. Theorem 2 below is known for commutative complex Banach algebras [4, p. 8]. Many devices introduced by Gelfand will be recognized in our proofs.

By a topological division algebra we mean one which possesses a unit 1 and a two-sided inverse $x^{-1}$ for each nonzero element $x$ in it. 
The following theorem is a convenient starting point.

THEOREM 1. Let $A$ be a convex complex topological division algebra with continuous inversion. Then $A$ consists only of scalar multiples of the unit element and is thus isomorphic with the complex number field.

Proof. If possible, select an element $x$ from $A$ which is not a scalar multiple of $1 \in A$. Since $A$ is convex, there exists a continuous, linear, real-valued functional $f$ defined on $A$ and such that $f\left(x^{-1}\right) \neq 0[10$, p. 162]. Define $g(z)=f(z)-i f(i z)$ for any $z \in A$. Then $g(\lambda z)=\lambda(g(z))$ for both real or complex $\lambda$. The function $h(\lambda)=g\left[(x-\lambda)^{-1}\right]$ is defined and continuous for any complex number $\lambda$ because $x$ is such that $x-\lambda \neq 0$ for any $\lambda$. From the relations

$$
\begin{aligned}
h(\mu)-h(\lambda) & =g\left[(x-\mu)^{-1}-(x-\lambda)^{-1}\right] \\
& =g\left[(x-\mu)^{-1}(x-\lambda-x+\mu)(x-\lambda)^{-1}\right] \\
& =(\mu-\lambda) g\left[(x-\mu)^{-1}(x-\lambda)^{-1}\right],
\end{aligned}
$$

we can deduce that $h(\lambda)$ has a derivative $h^{\prime}(\lambda)=g\left[(x-\lambda)^{-2}\right]$. Therefore $h$ is an entire function. Moreover, as $\lambda \rightarrow \infty$, we evidently have $h(\lambda) \rightarrow 0$, whence $h(\lambda) \equiv 0$. This contradicts $f\left(x^{-1}\right) \neq 0$. Therefore each $x$ in $A$ is a scalar multiple of the unit of $A$.

If the division algebra $A$ arises as the quotient algebra of a complex Banach algebra, one can easily verify the hypothesis of Theorem 1 .

The following considerations will enable us to drop the hypothesis that the topological algebra $A$ admit complex scalars.

Let us call $A$ formally real if $A$ is commutative and $x^{2}=-y^{2}$ in $A$ implies $x, y=0$. Let $A(i)$ be the class of all ordered pairs; and denote the pair $(0,1)$ by the symbol " $i$." Then each element of $A(i)$ can be written in the form $x+i y$; and algebraic operations can be defined in $A(i)$ in an obvious fashion such that $i^{2}=-1$. It is clear that complex scalars can be applied to $A(i)$. Let $U$ be a system of neighborhoods $U$ of 0 in $A$; then let us take the totality of sets $U+i U, U \in V$, as a system of neighborhoods for 0 in $A(i)$. One can readily verify that $A(i)$ is a complex topological algebra; that $A(i)$ is convex or complete if and only if $A$ is; that it is an integral domain or a field if and only if $A$ is; and that inversion is continuous in $A(i)$ if it is in $A$.

These concepts having been introduced, we proceed to the case in which only real scalars are required.

ThEOREM 2. Let $A$ be a convex topological division algebra with continuous inversion. The $A$ is isomorphic with either the real, the complex, or the quaternion field. 
Proof. Commutative case: If $A$ is formally real, then the construction of $A(i)$ can be carried out, yielding a convex, complex, topological algebra, with continuous inversion. By Theorem 1, $A(i)$ consists only of scalar pairs; therefore $A$ consists only of real multiples of the identity.

If $A$ is not formally real, let $x^{2}=-y^{2}$ with $x, y \neq 0$. Define $j=x y^{-1}$. Next, scalar multiplication by complex numbers $a+b i$ can be defined, by $(a+b i) z=a z+b(j z)$, for $z \in A$. Theorem 1 now tells us that $A$ is the complex number field.

General case: The center $Z$ of $A$ comes under the previous case, and is therefore the real or complex field. Let $x$ be selected outside of $Z$; and let $X$ be the division sub-algebra generated by $x$ over $Z$. Since this is still commutative, we may conclude that $x$ satisfies some polynomial with coefficients from $Z$ (and, in fact, of degree not greater than 2). Hence $A$ is a division algebra algebraic over the real number field. The argument is now completed by an appeal to the following lemma, whose proof can be conducted in the same way as that of Frobenius' theorem for algebras of finite order (dimension); or one may refer to a recent result [5, Theorem 16]:

LemMa. An algebraic division algebra over the real number field is either the real, the complex, or the quaternion field.

The requirement that inversion be continuous in $A$ is troublesome, although of course it is necessary. The most convenient way of insuring it is to suppose that $A$ can be given a norm having the property

$$
\|x y\| \leqq\|x\|\|y\|
$$

as well as the usual normed linear space properties. In the case of such an $A$ we can establish the continuity of inversion independently of completeness, as follows: let $x$ have an inverse $y$, and suppose $x+h$ has an inverse $y+k$. Then $(y+k)(x+h)=1,(y+k)(1+h y)=y$, $y h y+k+k h y=0, k=-k h y-y h y$, whence

$$
\|k\| \leqq \frac{\|y h y\|}{1-\|h y\|}
$$

if $\|h\|\|y\|<1$. Therefore we have the hypothesis of Theorem 2, with the following result:

Corollary 1 (Mazur [6, Theorem I]). Let $A$ be a normed division algebra whose norm has the property

$$
\|x y\| \leqq\|x\|\|y\| \text {. }
$$


Then $A$ is the real, the complex, or the quaternion field.

From this one can deduce the following corollary.

Corollary 2 ([6, Theorem II $])$. Let $A$ be a normed algebra in which $\|x y\|=\|x\|\|y\|$. Then $A$ is the real, the complex, or the quaternion field.

Proof. In the commutative case we can use a well known device $[9$, p. 246] to imbed $A$ in a field to which Corollary 1 or even Ostrowski's theorem [9, p. 257] may be applied. The noncommutative case is reduced to the commutative case by an application of the lemma as in Theorem 2.

A less trivial result on integral domains will be considered in the next section.

The other case in which inversion is continuous is that in which $A$ is a complete separable metric algebra, for then the set of nonzero elements forms a separable metric locally complete set which is a group with continuous multiplication, and, by $[7$, p. 881], inversion is continuous. Combining this with Theorem 2, we obtain the following.

ThEOREM 3. Let $A$ be a convex, separable, metric and complete division algebra. Then $A$ is isomorphic with either the real, the complex, or the quaternion field.

I have been informed that the complex commutative case of this theorem was obtained recently by Mr. B. Yood. Mr. Yood's proof also makes use of Montgomery's theorem.

3. Integral domains. An element $z$ of a topological ring $A$ is called a topological divisor of zero if there exists a closed set $F \subset A$ not containing 0 but such that 0 is a limit point of $z F$ or $F z$. We shall call an element singular if it has no inverse in a ring with unit and nonsingular, otherwise.

Silov [8] has proved that if $x$ is an element of a Banach algebra $A$ and if $\lambda_{0}$ is a frontier point of the set of scalars $\lambda$ for which $x-\lambda$ is singular, then $x-\lambda_{0}$ is a topological divisor of zero. Silov concludes from this the following:

Any complex Banach algebra satisfying the norm condition $k\|x\|\|y\| \leqq\|x y\| \leqq\|x\|\|y\|$ with some fixed positive $k$ is the complex number field.

The argument of [8] applied only to complex Banach algebras because conceivably the set of $\lambda$ for which $x-\lambda$ is singular might be void for all nonscalar $x$ in a Banach algebra. But if this were so, then each $x \neq 0$ in $A$ would be nonsingular, or $A$ would be a division 
algebra and its structure would be given by Theorem 3 . In somewhat greater detail: One can adapt the argument of [8] to show that if the set $S$ of singular elements has a frontier point $z$ in a normed algebra, then $z$ is a topological zero divisor. In a Banach algebra the set $S$ is always closed. Now the condition $k\|x\|\|y\| \leqq\|x y\|$ certainly precludes the existence of nonzero topological zero-divisors, hence $S$ consists of 0 alone and $A$ is a division algebra. We formulate this result as a theorem.

THEOREM 4. Let $A$ be a normed algebra in which $k\|x\|\|y\| \leqq\|x y\|$ $\leqq\|x\|\|y\|$ with a fixed positive $k$. Then $A$ is the real, the complex, or the quaternion number system.

It is possible to omit the condition that $A$ be complete, since $A$ can be completed without losing the norm condition.

The reader will observe that we have confined ourselves to normed integral domains in this section. This seems to be necessary as we can exhibit a complete convex complex algebra which has no topological divisors of zero, and is not a division algebra:

Let $A$ be the class of functions holomorphic on an open subset $G$ of the complex plane; and let this function space be given the k-topology. ${ }^{2}$ Then this complex linear algebra is (1) convex, (2) metrizable, (3) complete, (4) not normable, (5) it contains no topological zero divisors, and (6) the set of singular elements is open.

Proof. (1) is obvious. For (2), see [1, Theorems 7 and 12]. For (3) observe [1, Theorem 10] and the fact that a sequence of holomorphic functions can converge uniformly on an open set only to a holomorphic function. Now (4) will follow from (6) and the well known properties of Banach algebras to which we have alluded in Theorem 4. As regards (5), let $f, g_{n} \in A$ with $f \neq 0$ and $f g_{n} \rightarrow 0$ in the $k$-topology, of $A$. Let a closed bounded set $K \subset G$ be given. We can cover $K$ with a finite number of regions $G_{1}, \cdots, G_{n}$ whose boundaries lie in $K$ and such that $|f(z)|$ has a positive minimum $m$ on the point set $C$ formed by these boundaries. For $z \in C$ we evidently have

$$
\left|g_{n}(z)\right| \leqq m^{-1} \sup _{t \in C}\left|f(t) g_{n}(t)\right| \text {. }
$$

Since the right-hand side tends to zero as $n$ tends to infinity we have $g_{n} \rightarrow 0$ in the $k$-topology, using the principle of the maximum modulus. Hence $f$ is not a topological zero-divisor. To prove (6) suppose that $f$

${ }^{2}$ We have proposed this name for the topology usually introduced into such function spaces. For its definition, see $[1$, p. 481]. In it, convergence simply means uniform convergence on the bounded closed subsets of $\mathrm{G}$. 
has no inverse in $A$. This means simply that $f\left(z_{0}\right)=0$ for some $z_{0} \in G$. About $z_{0}$ we can describe a small circle $C$ lying within $G$, and on which $f$ has a positive minimum $m$. The set $V$ of all $h \in A$ for which $\sup _{z \in C}|h(z)|<m$ constitutes a neighborhood of 0 in $A$, in the $k$-topology. But by Rouché's theorem, if $h \in U$ then $f$ and $f+h$ have the same number of zeros within $C$. Thus $f+h$ has no inverse in $A$. This proves our assertions.

4. The continuity of ring operations. We have not required multiplication to be continuous in both factors simultaneously, as is customary in our topological algebras, because we did not need this property. Nevertheless, complete metric algebras have it. We append this section to consider such matters. For greater generality, define an $m$-ring as a ring whose elements form a metrizable topological group under addition, and whose product is continuous in each factor, under the topology.

THeOREM 5. Let $A$ be a complete m-ring. Then multiplication is continuous in both factors simultaneously. If the set of nonsingular elements is a separable $G_{\delta}$ and $A$ has a unit, then the process of inversion is continuous in this set.

PRoof. It will suffice to show that if $x_{n} \rightarrow 0$ and a neighborhood $U$ of 0 be given, then there is a neighborhood $V$ of 0 and an integer $n$ such that $x_{m} V \subset U$ for $m \geqq n$.

Since $A$ is a topological group under addition, we can find a neighborhood $W$ of 0 such that $W^{-}-W \subset U$. Let $A_{n}$ contain those $y \in A$ for which $x_{m} y \in W^{-}$if $m \geqq n$. Since right multiplication is continuous, the set $A_{n}$ is closed. Each $y \in A$ is contained in some $A_{n}$ because $x_{n} y \rightarrow 0$ and so $x_{m} y \in W$ for $m$ not less than some suitable $n$. Hence some $A_{n}$ contains an open set $V+y$ where $V$ can be selected as a neighborhood of 0 . If $V+y \subset A_{n}$ then $x_{m} V+x_{m} y \subset W^{-}$for $m \geqq n$. One may select $n$ greater if necessary so that $x_{m} y \in W$ if $m \geqq n$. Then $x_{m} V \subset W^{-}$ $-W$ for $m \geqq n$, as desired.

If the set $R$ of nonsingular elements is a $G_{\delta}$ it is obviously of the second category. If $R$ is also separable then the elements of $R$ form a separable space of the second category and also a group in which multiplication is continuous. By [7, Theorem 2], $R$ is a topological group and inversion is continuous on $R$.

In view of Theorem 5 it becomes natural to ask whether or not the nonsingular elements of a complete $m$-ring always form a topological group. We can give an example that this is not so. So far as we know, this point has never been mentioned before. 
The ring we exhibit ror this purpose is the intersection $L^{\omega}$ of all $L^{p}$-spaces based on the interval $[0,1]$, with $p \geqq 1$. We say $f_{n} \rightarrow 0$ in $L^{\omega}$ if and only if $f_{n} \rightarrow 0$ in each $L^{p}, p \geqq 1$. Thus topologized, $L^{\omega}$ is a complete convex $m$-algebra [2]. Now consider the following sequence of nonsingular elements of $L^{\omega}$ :

$$
f_{n}(t)=\left\{\begin{array}{cl}
1 / n & \text { for } 0 \leqq t \leqq 1 / n, \\
1 & \text { for } 1 / n<t \leqq 1,
\end{array}\right.
$$

$n=1,2, \cdots$. This sequence approaches the unit function $1,1(t) \equiv 1$, of $L^{\omega}$. But the sequence of inverses $f_{1}^{-1}, f_{2}^{-1}, \cdots$ does not approach 1 since

$$
\int_{0}^{1}\left(\bar{f}_{n}^{-1}(t)-1(t)\right)^{p} d t=\int_{0}^{1 / n}(n-1)^{p} d t=\frac{(n-1)^{p}}{n}
$$

tends to infinity as $n \rightarrow \infty$ and $p>1$. Hence the nonsingular elements do not form a topological group. In other words, inversion is not continuous on the set of nonsingular elements.

\section{BIBLIOGRAPHY}

1. R. Arens, A topology for spaces of transformations, Ann. of Math. vol. 47 (1946) pp. $480-495$.

2. - The space $L^{\omega}$ and convex topological rings, Bull. Amer. Math. Soc. vol. 52 (1946) pp. 931-935.

3. G. Birkhoff, A note on topological groups, Compositio Math. vol. 3 (1936) pp. 427-431. $3-24$.

4. I. Gelfand, Normierte Ringe, Rec. Math. (Mat. Sbornik) N.S. vol. 9 (1941) pp.

5. N. Jacobson, Structure theory for algebraic algebras, Ann. of Math. vol. 46 (1945) pp. 695-767.

6. S. Mazur, Sur les anneaux lineaires, C. R. Acad. Sci. Paris, vol. 207 (1938) pp. 1025-1027.

7. D. Montgomery, Continuity in topological groups, Bull. Amer. Math. Soc. vol. 42 (1936) pp. 879-882.

8. G. Silov, On the extension of maximal ideals, C. R. (Doklady) Acad. Sci. URSS. vol. 29 (1940) pp. 83-84.

9. B. L. van der Waerden, Moderne Algebra, vol. 1, Berlin, 1937.

10. J. Wehausen, Transformations in linear topological spaces, Duke Math. J. vol. 4 (1938) pp. 157-169.

InSTITUTE For Advanced Study 